\title{
In Vitro Grown Micro-Tissues for Cardiac Cell Replacement Therapy in Vivo
}

\author{
Raja Ghazanfar Ali Sahito ${ }^{\mathrm{a}} \quad$ Xiaowu Sheng ${ }^{\mathrm{b}}$ Martina Maass ${ }^{c} \quad$ Nelly Mikhael $^{\mathrm{a}}$ \\ Sarkawt Hamad ${ }^{a}$ Carlos O. Heras-Bautista ${ }^{a}$ Daniel Derichsweiler ${ }^{a}$ \\ Dimitry Spitkovsky ${ }^{a}$ Frank Suhr ${ }^{d} \quad$ Markus Khalile Konrad Brockmeier ${ }^{f}$ \\ Marcel Halbach ${ }^{c}$ Tomo Saric ${ }^{a}$ Jürgen Hescheler ${ }^{a}$ Benjamin Krausgrillc \\ Kurt Pfannkuche ${ }^{a, f}$ \\ aCenter for Physiology and Pathophysiology, Institute of Neurophysiology, Medical Faculty, University

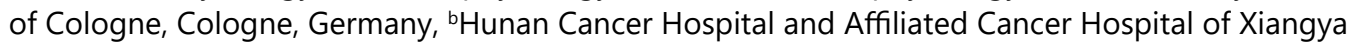 \\ School of Medicine, Central South University, Changsha, China, 'Department III of Internal Medicine,

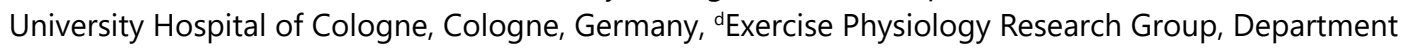 \\ of Movement Sciences, Group of Biomedical Sciences, KU Leuven, Leuven, Belgium, eDepartment for \\ Pediatric Cardiology, University of Giessen, Giessen, Germany, '́ Department for Pediatric Cardiology, \\ University Hospital of Cologne, Cologne, Germany
}

\section{Key Words}

Embryonic stem cells - Induced pluripotent stem cells - Thermo-responsive polymer • Regenerative medicine $\cdot$ Reconstructive medicine $\cdot$ Cardiac cell therapy $\bullet$ Cardiomyocytes

\footnotetext{
Abstract

Background/Aims: Different approaches have been considered to improve heart reconstructive medicine and direct delivery of pluripotent stem cell-derived cardiomyocytes (PSC-CMs) appears to be highly promising in this context. However, low cell persistence post-transplantation remains a bottleneck hindering the approach. Here, we present a novel strategy to overcome the low engraftment of PSC-CMs during the early post-transplantation phase into the myocardium of both healthy and cryoinjured syngeneic mice. Methods: Adult murine bone marrow mesenchymal stem cells (MSCs) and PSC-CMs were co-cultured on thermo-responsive polymers and later detached through temperature reduction, resulting in the protease-free generation of cell clusters (micro-tissues) composed of both cells types. Micro-tissues were transplanted into healthy and cryo-injured murine hearts. Short term cell retention was quantified by real-time-PCR. Longitudinal cell tracking was performed by bioluminescence imaging for four weeks. Transplanted cells were further detected by immunofluorescence staining of tissue sections. Results: We demonstrated that in vitro grown micro-tissues consisting of PSC-CMs and MSCs can increase cardiomyocyte retention by $>10$ fold one day post-transplantation, but could not fully rescue a further cell loss between day 1 and day 2. Neutrophil infiltration into the transplanted area was detected in healthy hearts

R. G. A. Sahito, X. Sheng, B. Krausgrill and K. Pfannkuche contributed equally to this work.

\begin{tabular}{ll}
\hline Kurt Pfannkuche & Center for Physiology and Pathophysiology, Institute of Neurophysiology, University of Cologne; Dept. III \\
and Benjamin Krausgrill & of Internal Medicine, University Hospital of Cologne, Cologne (Germany); Tel. +49 221478 6940, \\
& +49221478 96895, E-Mail kurt.pfannkuche@uni-koeln.de; benjamin.krausgrill@uk-koeln.de
\end{tabular}
}




\section{Cellular Physiology Cell Physiol Biochem 2019;52:1309-1324 \\ \begin{tabular}{ll|l} 
and BOI: 10.33594/000000092 & @ 2019 The Author(s). Published by \\
Cell Physiol Biochem Press GmbH\&Co. KG
\end{tabular} \\ Sahito et al.: Cardiomyocyte Transplantation}

and could be attributed to the cellular implantation rather than tissue damage exerted by the transplantation cannula. Injected PSC-CMs were tracked and successfully detected for up to four weeks by bioluminescence imaging. Conclusion: This approach demonstrated that in vitro grown micro-tissues might contribute to the development of cardiac cell replacement therapies.

(c) 2019 The Author(s). Published by

Cell Physiol Biochem Press GmbH\&Co. KG

\section{Introduction}

In contrast to more primitive vertebrates, the mammalian heart exhibits only a minor capability to regenerate damaged myocardial tissue. Adult cardiomyocytes show no or negligible proliferation potential, while the contribution of c-kit ${ }^{+}$cardiac stem cells to cardiomyocyte turnover is still under debate [1-3]. The state-of-the-art in cardiomyocyte regeneration was recently summarized in form of a consensus paper by leading experts in the field [4].

Therefore, it has been postulated that "cell replacement therapy" through the direct delivery of in vitro grown cells by intramyocardial injection [5], intracoronary infusion [6] or transplantation of engineered cardiac muscle patches $[7,8]$ could present an attractive alternative strategy to replace damaged cardiac tissue and improve cardiac function under pathophysiological conditions. Proof-of-principle studies demonstrated that transplanted cardiomyocytes can engraft into the ventricular walls of recipient rodents and improve function of ischemically damaged hearts $[9,10]$. Tachiban et al. have partially attributed the cardiac functional improvement observed in their study to proangiogenic and anti-apoptotic paracrine effects generated by the transplanted cardiomyocytes, thus further highlighting that mechanisms by which cell transplantation enhances the functional parameters of ischemically injured hearts remain to be elucidated [11].

To date, it has not been finally established which transplantable cell types possess the highest potential to cure the failing heart. Transplantation of lineage-committed cardiomyocytes could be beneficial since these cells are ready to adopt their function in the cardiac tissue. A pioneering study by Chong and colleagues demonstrated that human embryonic stem cell-derived cardiomyocytes (hES-CMs) are capable of engrafting and electrically integrating into non-human primate cardiac tissue; however, the efficiency of cell engraftment remains unclear [12].

Co-transplanted bone marrow-derived mesenchymal stromal cells (MSCs) may have the potential to enhance the early engraftment of transplanted cardiomyocytes to the tissue because they are highly adhesive to matrix proteins. Moreover, MSCs were reported to increase the number of endogenous c-kit ${ }^{+}$cardiac stem cells almost 20 -fold when transplanted in a porcine model of cardiac cell replacement therapy, and might therefore function as a trigger of endogenous repair mechanisms [13]. Co-transplantation of MSCs together with c-kit ${ }^{+}$cardiac stem cells further enhanced scar size reduction when compared to the effect exerted by the transplantation of monocultures of either c-kit ${ }^{+}$cardiac stem cells or MSCs alone [14]. An early study by Min and colleagues indicates that transplanted MSCs improve cardiac function in pigs after myocardial infarction. The effect was further enhanced by co-transplantation of primary fetal cardiomyocytes [15].

Previously, we analyzed the effect of murine bone marrow-derived MSCs on the morphological integration of purified induced pluripotent stem cell-derived cardiomyocytes (iPS-CMs) into vital and non-vital cardiac tissue in vitro and proved a supporting effect of MSCs with respect to morphological and electrical integration of pluripotent stem cell-derived cardiomyocytes (PSC-CMs) [16]. Furthermore, the choice of MSCs for cotransplantation is supported by studies showing beneficial immunomodulatory effects of MSCs $[17,18]$. Heart protective paracrine effects of MSCs were enhanced when MSCs were cocultured with cardiomyocytes and had the chance to communicate with the cardiomyocytes by tunneling nanotubes [19]. As a result, the MSCs adjusted their secretome and increased 


\section{Cellular Physiology Cell Physiol Biochem 2019;52:1309-1324 \\ \begin{tabular}{l|l} 
and Biochemistry Published online: 4 May 2019 & $\begin{array}{l}\text { C } 2019 \text { The Author(s). Published by } \\
\text { Cell Physiol Biochem Press GmbH\&Co. KG }\end{array}$
\end{tabular} \\ Sahito et al.: Cardiomyocyte Transplantation}

the expression of pro-angiogenic factors. Furthermore, exosomes from MSCs were found to protect cardiomyocytes from oxidative stress [20]. Taken together, we identified a rationale in considering MSCs inclusion in cardiac cell replacement therapies.

Despite several previous studies demonstrating a strong functional improvement upon cell transplantation to the injured myocardium, the limited efficiency of cell engraftment remains a major bottleneck for successful cardiac cell replacement therapy [9, 21, 22]. The process of cell transplantation and integration remains poorly understood; quantitative data on the initial persistence of transplanted cardiomyocytes is limited and the reasons for low engraftment rates remain obscure. This lack of knowledge hinders the development of more robust methods of cardiac cell therapy.

The major aim of this study was to evaluate the effect of co-transplantation of MSCs and PSC-CMs as preformed micro-tissues into healthy and acutely injured myocardium with respect to cardiomyocyte retention.

\section{Materials and Methods}

Culture of pluripotent stem cells

For cardiac differentiation, the male murine ES cell line $\alpha$ PIG44 [21] and the male murine iPS cell line FLuc- $\alpha$ PIG-AT25 [22] were used. The $\alpha$ PIG44 line is based on D3 mES cells and carries a reading frame for puromycin-N-acetyl-aminotransferase (PAC) and enhanced green fluorescent protein (eGFP) linked by an internal ribosome entry site under the control of the cardiac-specific alpha myosin heavy chain promoter [21]. The FLuc- $\alpha$ PIG-AT25 iPS cell line possesses the same cardiomyocyte specific transgene expressing eGFP and PAC as $\alpha$ PIG44. Additionally, FLuc- $\alpha$ PIG-AT25 iPS cells express firefly luciferase from an ubiquitin-C promoter knocked into the ROSA26 locus. For details of the generation of this cell line see Lepperhof et al. [22].

Pluripotent stem cells were cultured on mitotically inactivated murine embryonic fibroblasts in Dulbecco's Modified Eagle Medium (DMEM) supplemented with 15\% fetal calf serum (FCS), non-essential amino acids (1x), penicillin and streptomycin, $100 \mu \mathrm{M}$ 2-mercaptoethanol and $1000 \mathrm{units} / \mathrm{mL}$ leukemia inhibitory factor (LIF). All reagents were purchased from Thermo Fisher Scientific (Germany) except LIF (ORF Genetics, Kopavogur, Iceland). Cells were subcultivated every 2-3 days by trypsin digestion.

\section{Generation, culture and characterization of MSCs}

MSCs were generated from 6-8 week-old 129S2 male mice. MSC for cluster adhesion experiments (Fig. $1 \mathrm{H}, \mathrm{I}$ ) were generated from mice line (ROSA $\mathrm{mT} / \mathrm{mG}$ ) constitutively expressing the red fluorescent protein Tomato in all tissues. To prepare primary cultures the femur bones were flushed with DMEM using a syringe with injection cannula to extract the bone marrow. Bone marrow was repeatedly sucked through the cannula in order to prepare a cell suspension. The cells were plated on $10 \mathrm{~cm}$ culture dishes in high glucose (4.5 g/L) DMEM with 10\% FCS and $6 \mathrm{ng} / \mathrm{mL}$ basic fibroblast growth factor (bFGF, PeproTech, Hamburg, Germany). Cells were plated and non-adherent cells were removed after 12 hours. In the first 3 days following preparation medium was changed daily. Cells were further cultured until highly proliferative colonies of adherent cells occurred. These cells were routinely subcultured once they reached about $80 \%$ confluency. From the second passage onwards MSCs were cultured in low glucose (1 g/L) DMEM with 10\% FCS and $6 \mathrm{ng} / \mathrm{mL}$ bFGF.

For flow cytometric analysis MSC cultures (batch 1 passage 11, batch 2 passages 29 and 30, batch 3 passages 40 and 41) on $10 \mathrm{~cm}$ dishes were washed by PBS without $\mathrm{Ca}^{2+}$ and $\mathrm{Mg}^{2+}$ and dissociated into single cells by $0.05 \%$ trypsin/EDTA for 10 minutes. $0.25 \times 10^{6}$ MSCs per analysis were transferred into a $1.5 \mathrm{~mL}$ Eppendorf tube, washed by FC buffer containing $0.5 \%$ bovine serum albumin (BSA) (Sigma-Aldrich) and $0.1 \mathrm{mM}$ EDTA (Sigma-Aldrich) in PBS, and centrifuged at $300 \mathrm{~g}$ for 2 minutes. Surface marker staining was performed by re-suspending the cell pellet in $50 \mu \mathrm{L} \mathrm{FC} \mathrm{buffer} \mathrm{containing} \mathrm{1:10} \mathrm{dilutions} \mathrm{of} \mathrm{anti-CD90-}$ PE, anti-CD45-PE, anti-CD73-PE, anti-CD29-PE, anti-CD11b-PE-vio770, anti-Sca-1-vioBright ${ }^{\mathrm{TM}}$-FITC, and antiCD140b-APC for 10 minutes at $4{ }^{\circ} \mathrm{C}$. All antibodies were purchased from Miltenyi Biotech (Bergisch Gladbach, Germany). Next, $0.5 \mathrm{~mL} \mathrm{FC}$ buffer was added per tube, centrifuged at $300 \mathrm{~g}$, and the cell pellet was 


\section{Cellular Physiology and Biochemistry}

Cell Physiol Biochem 2019;52:1309-1324

Fig. 1. Generation of in vitro grown micro-tissues for cardiac cell replacement therapy. Microtissues were generated on thermoresponsive polymer surfaces. Cell detachment resulted in the formation of aggregates of MSCs and ES-CMs. For low-dose MSC/ ES-CM experiments, optimized ratios of MSCs and ES-CMs (green) were plated (A). Micro-tissues formed under low-dose MSC/ES$\mathrm{CM}$ conditions by cold-induced detaching are shown (CMs: green, MSCs: red) (B,C). Micro-tissues that were passed through an injection cannula readily attached to conventional tissue culture surfaces (D). Coating of thermoresponsive polymer surfaces

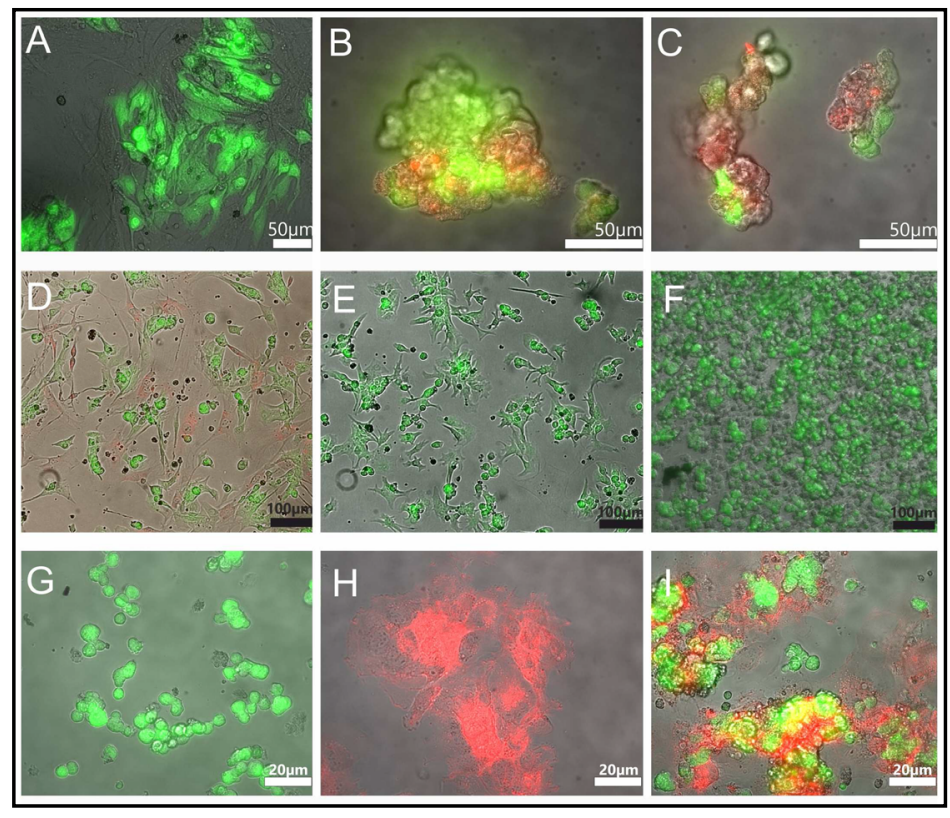
with fibronectin allowed for the formation of micro-tissues from pure ES-CMs without addition of MSCs (E) and could be detached from the surface of the culture dish and re-plated after passing the injection cannula without affecting cell integrity (F). iPS-CMs lifted from thermo-responsive polymers were cultured for 2 hours on tissue culture plastics and did not show signs of adhesion (G). MSCs prepared from the bone marrow of red fluorescent protein (Tomato) expressing mice, cultured for 2 hours on tissue culture plastics, adhered well (H). Micro-tissues composed of iPS-CMs and MSCs plated for 2 hours on tissue culture plastic adhered well and anchored the iPS-CMs to the surface (I). Note that the MSCs were stained by CMTPX (red fluorescence) in images B, C, and D. Cardiomyocytes express eGFP and display green fluorescence (all panels).

re-suspended into $0.250 \mathrm{~mL} \mathrm{FC} \mathrm{buffer,} \mathrm{and} \mathrm{maintained} \mathrm{at} 4{ }^{\circ} \mathrm{C}$ until assayed by flow cytometer (LSR Fortessa Analyzer, BD Biosciences). Data were analyzed by FCS express 6 (De Novo Software, Glendale, CA).

\section{Staining of MSCs with Celltracker CMPTX dye}

In some experiments MSCs were stained with the red fluorescent live cell stain CellTracker ${ }^{\mathrm{TM}}$ Red CMTPX Dye (Invitrogen Life Technologies) in accordance with manufacturer's protocols. In brief, the cells were incubated with $5 \mu \mathrm{M}$ CMTPX dye dissolved in MSC culture medium for 45 minutes. Following staining the cells were washed twice with PBS.

\section{Cardiac differentiation of PSCs and purification of cardiomyocytes}

In order to induce cardiac differentiation, PSCs were harvested as a single cell suspension. Cells were washed in calcium and magnesium-free PBS and trypsin $(0.05 \%)$ / EDTA $(0.02 \%)$ was added for approximately 5 minutes at $37^{\circ} \mathrm{C}$. The reaction was stopped by addition of FCS-containing medium and the cells were repeatedly passed through a $1000 \mu \mathrm{l}$ pipette tip to form a single cell suspension. Finally, the cells were resuspended at a concentration of $10^{5} \mathrm{cells} / \mathrm{mL}$ in cell culture medium. The medium used for differentiation was Iscove's Modified Dulbecco's Medium (IMDM) supplemented with 20\% FCS and ascorbic acid (L-Ascorbic Acid Phosphate Magnesium Salt n-Hydrate, Wako Chemicals GmbH, Neuss, Germany) at a concentration of $100 \mu \mathrm{g} / \mathrm{mL}$ during embryoid body (EB) formation and differentiation. The cell suspension was agitated on a rocking table inside a cell culture incubator to initiate formation of EBs. On day 2, EBs were counted and diluted at 200 EBs per dish $(10 \mathrm{~cm}$ ) in $14 \mathrm{~mL}$ medium (same composition as above including ascorbate).

On day 9, the medium was exchanged and no further ascorbate was added. Puromycin (Invivogen, San Diego, CA, USA) was applied at a concentration of $10 \mu \mathrm{g} / \mathrm{mL}$ in order to erase any non-myocyte and enrich pure PSC-CMs. The medium was changed every other day. 


\section{Cellular Physiology Cell Physiol Biochem 2019;52:1309-1324 \\ \begin{tabular}{ll|l} 
and Biochemistry $10.33594 / 000000092$ & $\begin{array}{l}\text { O } 2019 \text { The Author(s). Published by } \\
\text { Cell Physiol Biochem Press GmbH\&Co. KG }\end{array}$ \\
\cline { 2 - 3 }
\end{tabular} \\ Sahito et al.: Cardiomyocyte Transplantation}

Detailed characterization and assessment of cardiomyocyte purity of $\alpha$ PIG44-ESC-derived CMs and FLuc- $\alpha$ PIG-AT25 iPSC-derived CMs are found in [21-24].

\section{Generation of in vitro grown micro-tissues}

Co-culture of ES-CMs and MSCs was performed on 6-well-cell culture dishes coated with the thermoresponsive polymer Poly( $N$-isopropylacrylamide) (PNIPAAm) commercially available as "UpCell dishes" manufactured by Nunc and provided by VWR (Darmstadt, Germany). In order to generate micro-tissue, cell seeding was performed as follows: On day $11 \times 10^{5}$ MSCs (high-dose MSC condition) or $0.5 \times 10^{5}$ MSCs (lowdose MSC condition) were plated per individual well in UpCell 6-well dishes. The wells were not coated by any protein to prevent cardiomyocytes from adhesion to the polymer (PSC-CMs will hardly adhere to the polymer surface without coating by fibronectin or laminin). Day 2: Proper growth of MSC was assessed by phase-contrast microscopy. Cardiomyocytes (day 13 of differentiation) were dissociated by $0.05 \%$ trypsin/EDTA (Invitrogen) for about 30 minutes. We used young cardiomyocytes because previous data of our group indicates a loss of cardiomyocyte quality with increasing culture duration [25]. The resulting cell suspension was passed through a $40 \mu \mathrm{M}$ cell strainer (Falcon BD) to remove cell clumps and enable accurate cell counting. $5 \times 10^{5}$ ES-CMs (high dose MSC/ES-CM condition) or 8x10 5 ES-CMs (low dose MSC/ ES-CM condition) were plated per well of the previously MSC-attached 6-well UpCell dish. Day 4: Culture vessels were incubated on ice for 30 minutes to initiate cell detachment and the resulting micro-tissues were collected. Cell preparations were subjected to centrifugation for 1 minute at $180 \mathrm{x}$ g. Following supernatant removal, the cells were washed with PBS and settled by centrifugation. The resulting pellet was resuspended in $20 \mu \mathrm{L}$ DMEM and stored on ice until transplantation.

Generation of in vitro grown micro-tissues of pure cardiomyocytes

UpCell dishes were coated with fibronectin at a concentration of $0.125 \mu \mathrm{g} / \mathrm{mL}$ PBS (fibronectin from bovine plasma, sterile solution, Sigma Aldrich) for at least 1.5 hours at $37^{\circ} \mathrm{C}$. ES-CMs at day 13 of differentiation were dissociated by $0.05 \%$ trypsin/EDTA (Invitrogen) and plated at a density of $0.5 \times 10^{6}$ cells per well. After 3 days of culture, UpCell plates were chilled on ice and micro-tissues were collected as described before. Integrity of the micro-tissues was analyzed by phase contrast microscopy prior to transplantation.

Open chest surgery and cell transplantation

Transplantation protocols were approved by the local animal welfare committee and confirmed according to animal care law for laboratory animals. In this study, 6-8 week-old female wild-type mice were used (Strain 129S2/sv for ES cell experiments and F1 animals bred from 129S2/sv and C57BL/6j lines for iPS cell experiments to match the genetic background). Transplantation was performed as previously described [26]. General anesthesia was induced and maintained by inhalation of 1:1 mixture of nitrous oxide $\left(\mathrm{N}_{2} \mathrm{O}\right)$ and oxygen with gradual addition of initially $5 \%$ followed by $1.5 \%$ isoflurane (Baxter, Germany). After lateral thoracotomy, cells were either directly injected into the apical free lateral wall of the left ventricle (healthy heart) or injected following cryo-injury. Cryo-injury was induced by applying a liquid nitrogen cooled copper probe to the anterior LV free wall in 3 intervals of freezing for 5 seconds each. We chose the cryo-injury model for cell injection because coronary artery ligation results in an increased variability of the size of the lesion. Furthermore, coronary artery ligation produces infarctions of very large sizes in mice. In larger animals, coronary artery ligation or balloon catheter driven coronary artery occlusion are the method of choice and will be evaluated in the next experimental stage.

In order to minimize the variability of cell numbers injected per experiment, cells for individual injections were stored as aliquots in $20 \mu \mathrm{l}$ medium and briefly mixed prior to intake into the injection cannula. Per experiment, $20 \mu \mathrm{l}$ of cell suspension were applied at two adjacent transplantation sites by two injections of $10 \mu \mathrm{l}$ each by using a Hamilton syringe (H. Faust GmbH, Rheinbach, Germany) attached to a 29-gauge needle (Supplementary Fig. S1 - for all supplemental material see www.cellphysiolbiochem.com). After intramyocardial cell injection, the intercostal space was sutured and the skin was clapped with pins or sutured (for bioluminescence imaging). Isoflurane was withdrawn and replaced by high oxygen for the mice to emerge from sedation. 


\section{Cellular Physiology Cell Physiol Biochem 2019;52:1309-1324 \\ \begin{tabular}{ll|l} 
DOl: 10.33594/000000092 & $\begin{array}{l}\text { O } 2019 \text { The Author(s). Published by } \\
\text { Cell Physiol Biochem Press GmbH\&Co. KG }\end{array}$
\end{tabular} \\ Sahito et al.: Cardiomyocyte Transplantation}

\section{Genomic DNA preparation and quantitative real time TaqMan-PCR}

Explanted recipient hearts were stored at $-20^{\circ} \mathrm{C}$ for real-time PCR analysis. Preparation of genomic DNA and cell quantification by quantitative real time TaqMan-PCR were performed as described previously $[27,28]$. Genomic DNA was isolated from all samples using the DNeasy-Blood-and-Tissue-Kit from Qiagen. The retained fraction of transplanted male cells (mES-CM and MSCs) and transplanted transgenic cells (only mES-CM) within the female wild-type recipient hearts' ventricles was determined using quantitative real time TaqMan-PCR (7300 Real time PCR System, Applied Biosystems) according to manufacturer protocols with TaqMan ${ }^{\circledR}$ Gene Expression Master Mix (Applied Biosystems) and primers specific for Y-chromosome (SRY: Mm00441712_s1, Applied Biosystems), for the puromycin N-acetyl-aminotransferasetransgene (PAC: Custom TaqMan Gene Expression Assays on sequence

ATGACCGAGTACAAGCCCACGGTGCGCCTCGCCACCCGCGACGACGTCCCCAGGCCGTACGCACCCTCG

CCGCCGCGTTCGCCGACTACCCCGCCACGCGCCACACCGTCGATCCGGACCGCCACATCGAGCGGGTCAC

CGAGCTGCAAGAACTCTTCCTCACGCGCGTCGGGCTCGACATCGGCAAGGTGTGGGTCGCGGACGACGG

CGCCGCGGTGGCGGTCTGGACCACGCCGGAGAGCGTCGAAGCGGGGGCGGTGTTCGCCGAGATCGGCCC

GCGCATGGCCGAGTTGAGCGGTTCCCGGCTGGCCGCGCAGCAACAGATGGAAGGCCTCCTGGCGCCGCA

CCGGCCCAAGGAGCCCGCGTGGTTCCTGGCCACCGTCGGCGTCTCGCCCGACCACCAGGGCAAGGGTCTG

GGCAGCGCCGTCGTGCTCCCCGGAGTGGAGGCGGCCGAGCGCGCCGGGGTGCCCGCCTTCCTGGAGACCT

CCGCGCCCCGCAACCTCCCCTTCTACGAGCGGCTCGGCTTCACCGTCACCGCCGACGTCGAGGTGCCCGA

AGGACCGCGCACCTGGTGCATGACCCGCAAGCCCGGTGCCTGA, Applied Biosystems) and for beta-actin as house-keeping gene (BACT: Mm00607939_s1, Applied Biosystems).

\section{In vivo imaging}

Imaging of bioluminescent signals was performed on an IVIS 200 system (Xenogen, Caliper Life Sciences). The mice were injected with $300 \mathrm{mg} / \mathrm{kg}$ D-luciferin in PBS intraperitoneally. Ten minutes after injection the mice were anesthetized by $2.5 \%$ isoflurane and transferred to the imaging system. Data was collected for 60 seconds at high binning. Image analysis was performed with the software "Living Image 3D", version 2.5.1.

\section{Histology}

Explanted hearts were fixed in $4 \%$ paraformaldehyde (PFA) in PBS overnight at $4^{\circ} \mathrm{C}$. Samples were transferred into calcium- and magnesium-free PBS on the following day. Fixed samples were embedded in TissueTek O.C.T. (Sakura Finetek Europe B.V., Alphen aan den Rijn, Netherlands), frozen at $-80^{\circ} \mathrm{C}$ and cut into slices of $8 \mu \mathrm{m}$ thickness on a cryotome (Leica, Wetzlar, Germany). Trichrome stainings were performed with a Masson-Goldner-Trichrome staining kit according to manufacturer's instructions (Carl Roth, Karlsruhe, Germany).

For immunohistochemical stainings, heart slices were incubated in 10\% Roti-ImmunoBlock (Carl Roth) to block non-specific protein-protein interactions for 1-2 hours at RT. In order to detect neutrophils, heart slices were incubated with purified anti-neutrophil antibodies clone 7/4 (Cat. no. CL8993AP, Cedarlane, Burlington, USA) at a concentration of 1:200 overnight at $4^{\circ} \mathrm{C}$. The secondary antibody (1:1000 dilution in 1\% Roti-ImmunoBlock in PBS) Alexa Fluor 647 goat anti-rat IgG $(\mathrm{H}+\mathrm{L})$ (Thermo Fisher Scientific) together with the nuclear stain Hoechst 33342 at a concentration of $1 \mu \mathrm{g} / \mathrm{mL}$ was incubated for 1 hour at room temperature.

To detect transplanted FLuc- $\alpha$ PIG-AT25 iPSC-CMs heart slices were incubated with rabbit polyclonal antibodies against firefly-luciferase (Abcam, Cambridge, UK). The secondary antibody (1:1000 dilution in 1\% Roti-ImmunoBlock in PBS) Alexa Fluor 680 goat anti-rabbit IgG $(\mathrm{H}+\mathrm{L})$ (Thermo Fisher Scientific) together with the nuclear stain Hoechst 33342 at a concentration of $1 \mu \mathrm{g} / \mathrm{mL}$ was incubated for 1 hour at room temperature.

Fluorescent and histological images were taken using the Axiovert 200 fluorescence microscope and AxioVision 4.5 software (Carl Zeiss, Jena, Germany). Confocal images where taken on a SP8 confocal microscope equipped with white light laser (Leica Microsystems, Wetzlar, Germany).

\section{Statistical analysis}

All data are presented as mean \pm SEM. Differences have been tested for statistical significance using Student's t-test or one way ANOVA with correction for multiple comparisons, where appropriate. 


\section{Results}

\section{Generation of transplantable micro-tissues}

Flow cytometric analysis of murine bone marrow derived MSCs revealed a surface marker expression of CD29 $9^{\text {high }}$, Sca $1^{\text {high }}$, CD140B ${ }^{\text {high }}, \mathrm{CD}^{\text {low }}$ (Supplementary Fig. 2). CD90 expression largely varied between the passages from $13.1 \%$ to up to $71.7 \%$. CD 45 expression was close to background and CD11b was undetectable.

Cardiomyocytes were generated from the murine ES cell line $\alpha$ PIG44 and purified by puromycin as described previously [21].

Temperature-responsive polymers allow for the detachment of cultured cells by reducing temperature for a short time span, thereby avoiding protease treatment of the cultures. We have successfully utilized this technique to generate micro-tissues which were subsequently injected into the murine ventricular myocardium. Based on the hypothesis that MSCs facilitate the attachment of transplanted cells, we combined purified male murine ES-CMs with male murine MSCs in micro-tissues (Fig. 1A and 2) at two different ratios (low dose MSC condition vs. high dose MSC condition) in order to improve the transplantation efficiency of ES-CMs.

Fluorescence microscopy of micro-tissues consisting of CMTPX-labeled MSCs and eGFP ${ }^{+}$ ES-CMs (low-dose MSC condition) clearly showed the presence of both cell types in individual micro-tissues before transplantation (Fig. 1B, C).

In order to rule out that the passage of micro-tissues through the thin injection needle impairs the quality of the preparation and mechanically destroys the cells, micro-tissues were taken up through the injection needle and plated onto a tissue culture plate. The passage of the micro-tissues through the thin injection cannula neither affected the ability of the microtissues to attach to conventional tissue culture surfaces nor disturbed cell viability (Fig. 1D).

\section{Control groups included in the study}

To compare the effect of mixed micro-tissues composed of MSCs and ES-CMs with pure ES-CMs, two control groups were included in the study. In the first control group single cell suspensions of ES-CMs were generated by trypsin digestion. This group serves as a baseline to identify an improved PSC-CM retention in our approach.

In the second control group micro-tissues from pure ES-CMs (Fig. 1E) were generated. This group was included to compare the effect of transplantation of micro-tissues composed of pure PSC-CMs versus PSC-CMs single cell suspensions as well as to determine whether MSCs inclusion in the micro-tissue would increase the retention of CMs. Again, passage of pure ES-CM micro-tissues through the injection cannula did not affect the ability of the cells to attach to fibronectin-coated plastic surfaces (Fig. 1F). It was hypothesized that micro-tissues combined of MSCs

and PSC-CMs

improve the cell retention by overcoming low matrix adhesion of PSC-CMs. To test this hypothesis the following experiment was performed: iPSCMs lifted from thermo-responsive polymer surfaces and replated onto tissue culture



Fig. 2. Principle of micro-tissue preparation from MSCs and ES-CMs on thermoresponsive polymers. 
plastics did not show signs of attachment even after 2 hours of culture (Fig. 1G). In contrast, red fluorescent MSCs were attached and well spread under the same conditions (Fig. 1H). Micro-tissues composed of red fluorescent MSCs and iPS-CMs replated for 2 hours on tissue culture plastics show strong matrix adhesion as well and anchored the cardiomyocytes to the surface (Fig. 1I).

\section{Short term retention of transplanted PSC-CMs in vivo}

In order to evaluate the potential benefit of micro-tissue preparation, four groups were analyzed in vivo: Group I (control) received monocultures of purified CMs in the form of single cell suspensions generated by trypsin digestion. Group II (control) received pure ESCM micro-tissues lifted from thermo-responsive dishes. Group III received high-dose MSCs/ ES-CM micro-tissues while Group IV received low dose MSC/ES-CM micro-tissues. Groups III and IV were included in the study to compare the effect of MSC addition in comparison to Group II.

To quantify the retention of transplanted cells in general and transplanted ES-CMs in particular, real-time-PCR was applied because this method enables precise quantifying of transplanted cells early after transplantation. The reason for this advantage is the fact that it is possible to standardize the quantification for the initial cell dose applied, while longitudinal approaches rely on a reference measurement after cell transplantation.

Healthy female wild-type mice were used as recipients, allowing us to quantify the total number of transplanted cells based on SRY gene located on the Y-chromosome. On the other hand, cardiomyocytes were particularly detected via quantification of the PAC transgene specifically expressed in these cells.

In an initial experiment, a series of hearts $(n=9)$ was collected for analysis immediately after injection of micro-tissues (conducted for group III) to approximate the cells loss during injection and determine the maximum achievable cell retention. Quantification of ES-CMs in these samples resulted in $23.4 \pm 4.4 \%(n=9)$ retention of ES-CMs. This result indicates that 3 out of 4 ES-CMs were already lost during the injection process. It might be speculated that the pressure inside the contracting tissue and a lack of sufficient space to take up the volume of the injected cells is a reason for this substantial initial cell loss and forces the cells through the injection channel out of the tissue. Furthermore, the extraordinary density of capillaries in the myocardium may also contribute to an initial cell loss by taking cells into the bloodstream because it cannot be avoided that capillaries are damaged during injection. To test this hypothesis on a qualitative level, we performed an experiment on a Langendorff heart preparation that was retrogradually perfused with Krebs-Ringer-Henseleit solution (Supplementary video 1). The transplantation process was simulated by injecting saline colored with blue food dye into the myocardium. A transport of the injected solution in the coronary vessels was observed within seconds after the injection, demonstrating the loss of injected material through damaged vessels. It can be speculated that cells are transported away from the injection site together with the injected solution.

Further hearts were collected 24 and 48 hours following cell injection to characterize the cell retention in the acute phase after injection. Twenty-four hours after transplantation only $0.6 \pm 0.2 \%(n=5)$ of ES-CMs transplanted as single-cell-suspension (Group I) were found to be retained in the tissue (Fig. 3). Pure ES-CM micro-tissues lifted from thermo-responsive polymers (Group II) gave similar low efficiency for cell retention with $0.5 \pm 0.4 \%(n=7)$ after 24 hours and $0.5 \pm 0.3 \%(n=7)$ after 48 hours.

In contrast, transplantation of mixed MSCs and ES-CMs micro-tissues resulted in the retention of $11.3 \pm 3.1 \%(\mathrm{n}=14)$ of ES-CMs for low-dose MSC/ES-CM micro-tissues ( $\mathrm{p}<0.05$ vs. ES-CM micro-tissues after $24 \mathrm{~h})$ and $4.9 \pm 2.2 \%(\mathrm{n}=11)$ of ES-CMs for high-dose MSC/ ES-CM micro-tissues after 24 hours. Quantification of total transplanted male cells (i.e. ESCMs+MSCs) resulted in $8.2 \pm 2.3 \%(n=14)$ and $8.6 \pm 2.7 \%(n=11)$ of cell retention in these two groups, respectively. Two days after transplantation, the ES-CM retention was reduced to $4.5 \pm 1.2 \%(\mathrm{n}=8)$ in low-dose MSC/ES-CM micro-tissues group ( $\mathrm{p}<0.01$ vs. ES-CM microtissues after $48 \mathrm{~h})$ and to $1.0 \pm 0.4 \%(\mathrm{n}=6)$ in high-dose MSC/ES-CM micro-tissues group 
Fig. 3. Transplantation of in vitro grown micro-tissues: Early cell retention. The fraction of cells remaining after direct intra-myocardial injection was measured by real time PCR. Detection of male cells ("SRY") indicates the fraction of MSCs and ES-CMs together; detection of the puromycin-acetyltransferasetransgene ("CM") specifically indicates the fraction of ES-CMs that was retained in the tissue 24 or 48 hours after transplantation. The cell number initially injected was set as $100 \%$ using a control where cells were directly added


to an explanted heart in a reaction tube and processed for DNA extraction.

( $\mathrm{p}<0.05 \mathrm{vs.}$ low-dose MSC/ES-CM after $48 \mathrm{~h}$ ). Total cell retention of male transplanted cells was $6.5 \pm 3.2 \%(n=8)$ and $3.7 \pm 0.9 \%(n=6)$ in these two experimental groups, respectively.

The integration and retention of cardiomyocytes after transplantation may vary from MSCs. To test this, the fraction of ES-CMs within the whole population of transplanted male cells in the tissue was estimated based on the real time PCR quantification of total male cells (by using SRY primers) and specific detection of ES-CMs (by using the PAC-specific primers) (Supplementary Fig. 3). $24 \mathrm{hrs}$ after transplantation the fraction of ES-CMs was determined as $73.4 \pm 13.4 \%$ vs. $38.6 \pm 8.0 \%$ for low-dose MSC/ES-CM and high-dose MSC/ES-CM, respectively. After $48 \mathrm{hrs}$, the fraction of ES-CMs decreased in these groups to $46.5 \pm 16.7 \%$ vs. $18.1 \pm 7.1 \%$, respectively. These results indicate that the number of transplanted ES-CMs decreases over time when compared to the number of MSCs.

The results above suggest that the low-dose MSC/ES-CM condition is favorable to support cardiomyocyte retention. In order to estimate the absolute numbers of cells that were transferred to the heart in low-dose MSC/ES-CM conditions, we seeded $0.5^{*} 10^{5}$ MSCs and $8^{*} 10^{5} \mathrm{CMs}$ as described for the transplantation experiment. Micro-tissues were lifted on day 4 from the plate and dissociated into single cells for counting. The total number of viable cells as estimated by trypan blue staining was determined as $7.62^{*} 10^{5} \pm 2.43^{*} 10^{5}$ cells $(n=7)$ per well, corresponding to the cell dose for one heart. In a second experiment only $0.5^{*} 10^{5}$ MSCs were seeded without addition of CMs. The number of viable MSCs in micro-tissues at day 4 was estimated to be $1,89 * 10^{5} \pm 0.13^{*} 10^{5}$ cells $(n=4)$. By subtracting the number of MSCs found in micro-tissues prepared from MSC-monocultures from the total number of viable cells found in low-dose MSC/ES-CM preparations, we conclude that the number of cardiomyocytes transferred per animal is approximately $5.7^{*} 10^{5}$ cells under low-dose MSC/ ES-CM conditions. Based on the ES-CM retention of $11.3 \%$ after 24 hours as determined by real time PCR, the absolute retention of cardiomyocytes can be approximated as $6.4 * 10^{4}$ at this time point.

\section{Longitudinal tracking of transplanted PSC-CMs in vivo}

We have defined a method to improve the delivery of PSC-CMs into healthy murine myocardium. However, several questions remain unsolved. Firstly, it is unclear whether the technology increases PSC-CM retention in a clinically more relevant model of cardiac injury. Secondly, a further technique should be employed to verify the positive results generated by real time PCR. Thirdly, it is of significant interest to track the transplanted cardiomyocytes over a longer period of time. 
To address these further questions, a model of cardiac cryoinjury was applied. A cooled copper stamp was used to generate a defined lesion by controlled freezing of the cardiac tissue. The iPS cell line FLuc- $\alpha$ PIG-AT25 was chosen for the study because it stably expresses firefly luciferase from a save harbor locus (ROSA26) as described in a previous study [22]. Puromycin selection of differentiated FLuc- $\alpha$ PIG-AT25 iPS cells yielded purified cardiomyocytes as illustrated by immunocytochemical staining for cardiac actinin (Supplementary Fig. 4).

Cells for transplantation were prepared in the form of micro-tissues according to the low-dose MSC/iPS-CM protocol described above. The cryolesion was prepared immediately prior to cell injection, and the cells were transplanted at the border zone of the acute lesion that was clearly identified by its pale appearance $(n=8)$. For comparison, transplantation of micro-tissues of low dose MSC/iPS-CM were performed on healthy hearts (without cryolesion, $\mathrm{n}=3$ ).

One day after cell transplantation the mice were imaged for bioluminescence for the first time. Intraperitoneal injection of D-luciferin elicited emission of bioluminescence from the transplanted cardiomyocytes (Fig. 4A). Data was collected for each individual animal on days 1, 4, 7, 14, 21 and 28 after transplantation. For quantitative analysis the signal intensity recorded on day 1 was defined as $100 \%$ initial signal strength and the signal recorded on the consecutive days were normalized to this starting value to allow for direct comparison of all results. Results are shown as percent signal strength for iPS-CMs transplanted into healthy hearts (blue line) or cryo-injured hearts (red line), respectively (Fig. 4B). The data points to a tendency of increased cell retention in injured myocardium with a cell retention of $70 \%$ at day 7 as compared to day 1 and a continuous decline at later time points.

To validate the results, hearts from cryo-injured animals were collected and tissue sections were prepared. Trichrome staining was used to identify the fibrotic area that results from the scarring process after cryo-injury (Fig. 5A and E). The transplanted cardiomyocytes

Fig. 4. Bioluminescence detection of transplanted iPS-CMs. Luciferaseexpressing cardiomyocytes were transplanted under low-dose MSC/iPS-CM condition. Mice were analyzed for luciferase activity over 28 days and luminescence was quantitatively recorded (A). The red line indicates the relative bioluminescence of cells transplanted into hearts following cryolesion, while the blue line illustrates the bioluminescence of cells injected into healthy recipients. Luminescence signals were defined as $\quad 100 \% \quad$ (starting value) at day one after transplantation for each

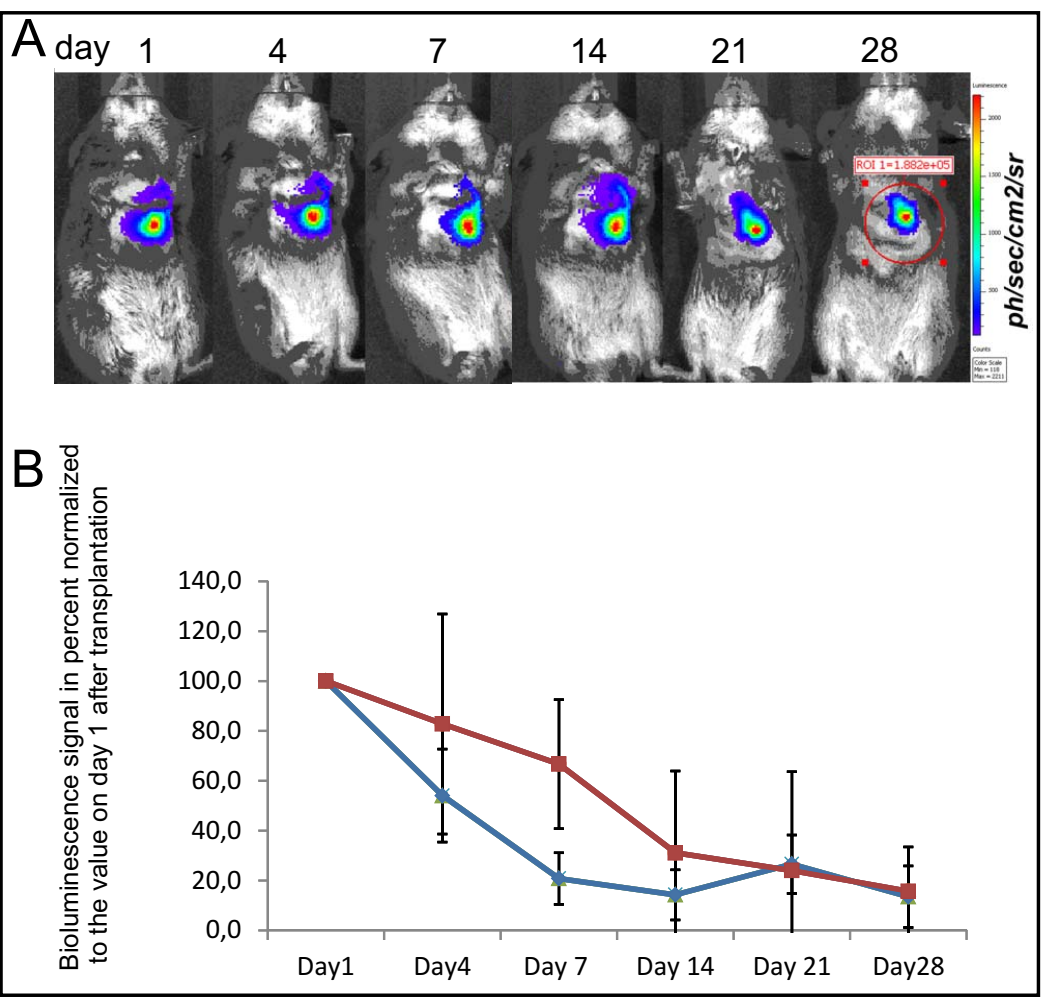
individual mouse. Relative luciferase signal as compared to the starting value at day one was calculated for each time point (B). 
were stained with antibodies against luciferase (shown in green). Fluorescence background was acquired to visualize the endogenous cardiomyocytes (shown in red). The cellular nuclei were stained by Hoechst dye (blue). Tissue samples were analyzed one week after transplantation. The sections shown in Fig. $5 \mathrm{~A}$ and $5 \mathrm{~B}$ show consecutive sections in order to illustrate the position of the engrafted cells relative to the tissue lesion (Fig. 5A/B and Fig. 5C/D display sections of 2 different animals). A further heart was prepared for histological analysis two weeks after transplantation (Fig. 5E-H) and transplanted cells were found in the damaged tissue (boxed area in Fig. 5E corresponds to the immunofluorescence picture shown in Fig. 5F). At higher power magnification an orientated structure of the integrated cells can be detected and an alignment of the transplanted cells with the direction of the endogenous cardiomyocytes was found (Fig. $5 \mathrm{G}, 5 \mathrm{H})$.

Transplanted cells induce a primary innate immune reaction

In order to investigate the potential role of the innate immune system in the observed short-term loss of transplanted micro-tissues, we stained heart slices 24 hours post-injection of ES-CMs into healthy heart tissue $(n=2)$ with antibodies against neutrophil granulocytes. Stained heart showed total infiltration with neutrophils
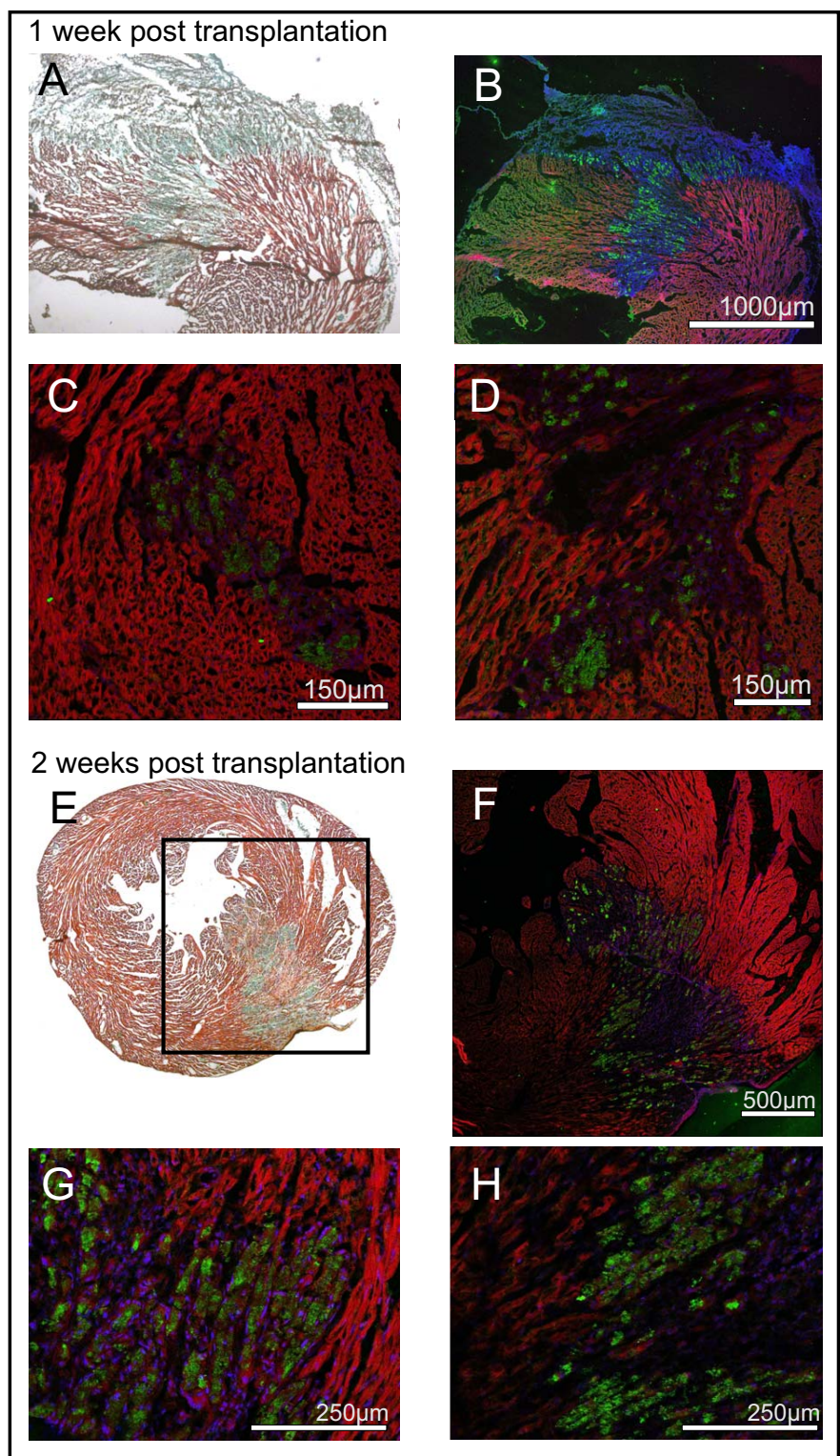

Fig. 5. Histological observation of transplanted micro-tissues. Micro-tissues of low-dose MSC/iPS-CM were transplanted into cryoinjured cardiac tissue. Trichrome staining revealed fibrotic areas of myocardial damage after one week (A) and two weeks (E). Transplanted cells were stained with antibodies against luciferase (green) and nuclei were co-stained by Hoechst dye (blue). Tissue autofluorescence (red) was recorded to visualize the endogenous cardiac tissue. Panel A/B and C/D show results for two individual animals one week after cryoinjury and cell transplantation. Panel E/F show engrafted cells from one animal two weeks after cell transplantation. Panel $\mathrm{G} / \mathrm{H}$ display magnified regions from the section shown in $\mathrm{F}$.

(Fig. 6), demonstrating its unique multi-lobed nucleus

(6B), particularly surrounding the micro-tissues. In order to exclude that the local injury from the injection needle was the reason for the observed neutrophil infiltration, heart slices injected with control PBS $(n=4)$ were stained at 0 and 24 hours post-transplantation with 
Fig. 6. Histological observation of neutrophil invasion. A tissue section from a healthy heart transplanted with ES-CMs (eGFP-expressing: green) and MSCs (red) was stained with antibodies against neutrophils (yellow). Nuclei were stained by Hoechst dye (blue). Arrows (right panel) point to multi-lobed nuclei.

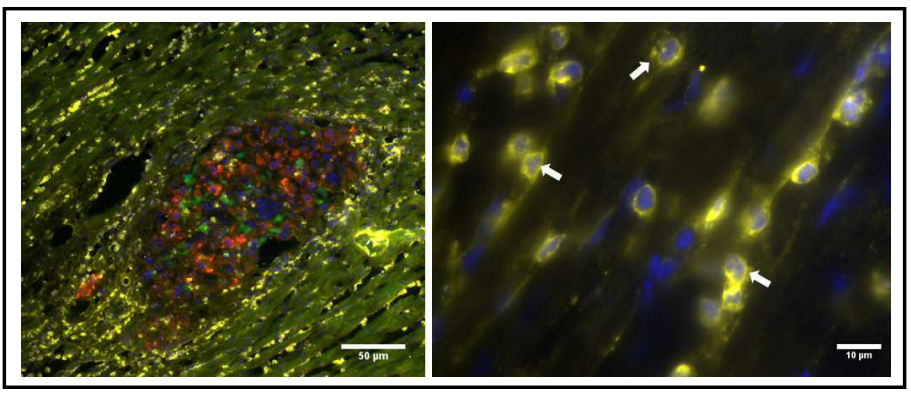

anti-neutrophil antibodies. Neutrophil invasion was absent in the injected hearts (data not shown) suggesting that the injected cells were the primary reason for the initiation of the observed primary immune reaction.

\section{Discussion}

The strategy of transplanting pre-formed micro-tissues composed of PSC-CMs and MSCs enhanced the early cell retention, whereas transplantation of pure PSC-CMs, either as single cells or micro-tissues resulted in very low cell engraftment below $1 \%$. These findings demonstrate the need for co-transplantation of non-cardiac cells for efficient delivery of PSCCMs into myocardial tissue, preferentially as pre-formed aggregates instead of mixed cell suspensions. Our data indicates that presence of MSCs in micro-tissues supports anchorage of transplanted cardiomyocytes. Omitting trypsin treatment and pre-formation of cell-matrix contacts is likely supportive in this regard.

MSCs used in this study were isolated from bone marrow by plastic adhesion. The cells were found to express CD29, an integrin receptor necessary for matrix adhesion, at high level as indicated by flow cytometry. The high expression of CD29 is in line with our assumption that MSCs support the matrix adhesion of micro-tissues composed of MSCs and CMs.

Very low expression of CD45 points to the absence of hematopoietic cells in our preparations. Missing expression of $\mathrm{CD} 11 \mathrm{~b}$ excludes contamination of the culture with monocytes or macrophages. Furthermore, Sca1 and CD140b were expressed at high level while CD73 was low and CD90 varied between different passages. The surface marker characteristics found here are in line with previous reports. CD90 expression of murine bone marrow MSCs was - in contrast to rat and human MSCs - identified to be low by Suto and colleagues [29]. Interestingly, Peister and colleagues found varying expressions of Sca1 in MSCs derived from different mouse strains and absence of CD90 on MSCs pointing to substantial variations in Sca1 and CD90 expression between various inbred strains [30].

The differences in ES-CM engraftment found between high-dose MSC/ES-CM and lowdose-dose MSC/ES-CM experiments in this study indicate that further optimization of the cell ratios, carefully determination of the optimal cell dose and fine-tuning of the methods might improve the cell retention results. This is underlined by the notion that the preparation of micro-tissues generated on thermo-responsive polymers is not yet standardized with respect to the cell number per individual micro-tissue.

Furthermore, it was found that the fraction of ES-CMs in the total population of transplanted cells decreased between $24 \mathrm{hrs}$ and $48 \mathrm{hrs}$ after transplantation (while the fraction of MSCs increased). This result could be due to a pronounced cell death of ES-CMs compared to MSCs or proliferation of the MSCs or a combination of both pathways and requires further analysis.

We found a reduction in cell persistence between $24 \mathrm{hrs}$ and $48 \mathrm{hrs}$ after transplantation and were able to detect an invasion of neutrophils to the site of cell transplantation in the intact heart. Invasion of neutrophils was not seen in animals that were only injected with PBS into the healthy myocardium. These data point to the assumption that factors released 


\section{Cellular Physiology Cell Physiol Biochem 2019;52:1309-1324

 \\ Sahito et al.: Cardiomyocyte Transplantation}

from injected cells rather than from the tissue that was mechanically injured by the injection attract neutrophils to the site of injury. It might be speculated that excessive activation of neutrophils plays a damaging role in the early phase of cell integration. Further elucidation of this pathway in a downstream study will help to separate supportive from damaging effects of innate immune cells in cell therapy.

Longitudinal tracking of transplanted iPS-CMs into healthy as well as cryo-injured hearts by a bioluminescence-based approach supported the conclusion that transplantation of micro-tissue is effective in delivering PSC-CMs to the site of cardiac injury. Transplantation into damaged myocardium even shows a tendency towards higher cell retentions one week after transplantation in cryo-injured tissue as compared to healthy tissue, indicating that the acute inflammatory process following tissue injury does not per se hinder the engraftment of injected PSM-CMs. Bioluminescence data of successful cell engraftment is supported by immunohistochemical detection of transplanted cardiomyocytes. Two weeks after transplantation, transplanted cells were found to align parallel to endogenous cardiomyocytes pointing to a mechanical integration into the tissue.

Successful intramyocardial transplantation of pluripotent stem cell-derived cardiomyocytes remains a challenging task and is likely affected by several independent factors. It is therefore important to define experimental settings that help to unravel the individual reasons for limited cell integration at distinct stages of the process. For example Chong and colleagues report a special suture technique (mattress suture) to entrap cells in the tissue [12]; this method is only applicable in large animal models but might further be improved in combination with the transplantation of in vitro grown micro-tissues. Another promising method that likely exerts synergistic effects is magnetic cell retention and has been tested by Cheng and colleagues in different approaches, resulting in a threefold increase in cell retention [31-33] when applied to cardiosphere-derived cells.

Ottersbach and colleagues recently described a method of magnetic retention that increases the retention of transplanted cardiomyocytes up to seven-fold but at low overall efficiency [34]. It appears likely that the magnetic retention did not uncover its full potential in these studies because magnetic retention alone is not sufficient to anchor the transplanted cells in the tissue. Our novel method of micro-tissue transplantation specifically addresses the issue of early phase cardiomyocyte adhesion to the host tissue and will generate additive effects when combined with magnetic retention in future approaches.

Moon and colleagues found an improved cardiac function when human ES-CMs were transplanted as micro-tissues of few (5-10) cells [35], supporting our conclusion that single cell transplantation is not appropriate to achieve robust cell engraftment. Moreover, Moon and colleagues demonstrate the formation of non-cardiac tissues, such as gland structures when human ES-CMs with a purity of approximately $90 \%$ were transplanted and, as a consequence, it cannot be ruled out that the remaining cells are responsible for the improved cell engraftment - possibly by the same mechanisms as described in this manuscript.

\section{Conclusion}

Thermo-responsive polymers are a powerful tool to prepare cells for cell replacement therapies. They allow for protease-free detachment of cells and facilitate the generation of micro-tissues at the same time. In our current approach, the early retention of ES-CMs was increased by more than 10-fold at the first day after transplantation when MSCs were coupled to the ES-CMs prior to transplantation. We are convinced that this approach can also be adapted to cell therapeutic applications other than cardiac cell replacement therapy. Future studies will develop strategies to generate micro-tissues of defined size and cell content and apply measures to limit the ultra-rapid cell loss during injection. 


\section{Acknowledgements}

This work was supported by grants from the "Else Kröner-Fresenius-Stiftung" (2012_ A143) to Kurt Pfannkuche and Benjamin Krausgrill, the "Dr--Robert-Pfleger-Stiftung" to Kurt Pfannkuche and the Imhoff Stiftung to Tomo Saric. We thank Cornelia Böttinger, Anette Peffekoven, Katja Urban and Ellen Veit for excellent technical assistance and the animal caretakers for their support. Confocal images were taken at the imaging facility of the Cologne Cluster of Excellence in Cellular Stress Responses in Aging-associated Diseases (CECAD). We thank Dr. Christian Jüngst for supporting us with imaging.

\section{Disclosure Statement}

The authors declare to have no competing interests.

\section{References}

- 1 van Berlo JH, Kanisicak O, Maillet M, Vagnozzi RJ, Karch J, Lin SC, Middleton RC, Marban E, Molkentin JD: c-kit+ cells minimally contribute cardiomyocytes to the heart. Nature 2014;509:337-341.

- 2 Vicinanza C, Aquila I, Scalise M, Cristiano F, Marino F, Cianflone E, Mancuso T, Marotta P, Sacco W, Lewis FC, Couch L, Shone V, Gritti G, Torella A, Smith AJ, Terracciano CM, Britti D, Veltri P, Indolfi C, Nadal-Ginard B, et al.: Adult cardiac stem cells are multipotent and robustly myogenic: c-kit expression is necessary but not sufficient for their identification. Cell Death Differ 2017;24:2101-2116.

3 Vagnozzi RJ, Sargent MA, Lin SJ, Palpant NJ, Murry CE, Molkentin JD: Genetic Lineage Tracing of Sca-1(+) Cells Reveals Endothelial but Not Myogenic Contribution to the Murine Heart. Circulation 2018;138:29312939.

4 Eschenhagen T, Bolli R, Braun T, Field LJ, Fleischmann BK, Frisen J, Giacca M, Hare JM, Houser S, Lee RT, Marban E, Martin JF, Molkentin JD, Murry CE, Riley PR, Ruiz-Lozano P, Sadek HA, Sussman MA, Hill JA: Cardiomyocyte Regeneration: A Consensus Statement. Circulation 2017;136:680-686.

5 Maass M, Krausgrill B, Eschrig S, Kaluschke T, Urban K, Peinkofer G, Plenge TG, Oeckenpohler S, Raths M, Ladage D, Halbach M, Hescheler J, Muller-Ehmsen J: Intramyocardially Transplanted Neonatal Cardiomyocytes (NCMs) Show Structural and Electrophysiological Maturation and Integration and DoseDependently Stabilize Function of Infarcted Rat Hearts. Cell Transplant 2017;26:157-170.

- 6 Johnston PV, Sasano T, Mills K, Evers R, Lee ST, Smith RR, Lardo AC, Lai S, Steenbergen C, Gerstenblith G, Lange R, Marban E: Engraftment, differentiation, and functional benefits of autologous cardiospherederived cells in porcine ischemic cardiomyopathy. Circulation 2009;120:1075-1083.

- 7 Gao L, Gregorich ZR, Zhu W, Mattapally S, Oduk Y, Lou X, Kannappan R, Borovjagin AV, Walcott GP, Pollard AE, Fast VG, Hu X, Lloyd SG, Ge Y, Zhang J: Large Cardiac Muscle Patches Engineered From Human InducedPluripotent Stem Cell-Derived Cardiac Cells Improve Recovery From Myocardial Infarction in Swine. Circulation 2018;137:1712-1730.

8 Wang Q, Yang H, Bai A, Jiang W, Li X, Wang X, Mao Y, Lu C, Qian R, Guo F, Ding T, Chen H, Chen S, Zhang J, Liu C, Sun N: Functional engineered human cardiac patches prepared from nature's platform improve heart function after acute myocardial infarction. Biomaterials 2016;105:52-65.

9 Laflamme MA, Chen KY, Naumova AV, Muskheli V, Fugate JA, Dupras SK, Reinecke H, Xu C, Hassanipour M, Police S, O'Sullivan C, Collins L, Chen Y, Minami E, Gill EA, Ueno S, Yuan C, Gold J, Murry CE: Cardiomyocytes derived from human embryonic stem cells in pro-survival factors enhance function of infarcted rat hearts. Nat Biotechnol 2007;25:1015-1024.

10 van Laake LW, Passier R, Doevendans PA, Mummery CL: Human embryonic stem cell-derived cardiomyocytes and cardiac repair in rodents. Circ Res 2008;102:1008-1010.

- 11 Tachibana A, Santoso MR, Mahmoudi M, Shukla P, Wang L, Bennett M, Goldstone AB, Wang M, Fukushi M, Ebert AD, Woo YJ, Rulifson E, Yang PC: Paracrine Effects of the Pluripotent Stem Cell-Derived Cardiac Myocytes Salvage the Injured Myocardium. Circ Res 2017;121:e22-e36. 


\section{Cellular Physiology Cell Physiol Biochem 2019;52:1309-1324 \\ \begin{tabular}{ll|l} 
and Biochemistry $\begin{array}{l}\text { DOl.33594/000000092 } \\
\text { Published online: } 4 \text { May 2019 }\end{array}$ & $\begin{array}{l}\text { O } 2019 \text { The Author(s). Published by } \\
\text { Cell Physiol Biochem Press GmbH\&Co. KG }\end{array}$ \\
\cline { 2 - 3 }
\end{tabular}}

Sahito et al.: Cardiomyocyte Transplantation

- 12 Chong JJ, Yang X, Don CW, Minami E, Liu YW, Weyers JJ, Mahoney WM, Van Biber B, Palpant NJ, Gantz JA, Fugate JA, Muskheli V, Gough GM, Vogel KW, Astley CA, Hotchkiss CE, Baldessari A, Pabon L, Reinecke H, Gill EA, et al.: Human embryonic-stem-cell-derived cardiomyocytes regenerate non-human primate hearts. Nature 2014;510:273-277.

- 13 Hatzistergos KE, Quevedo H, Oskouei BN, Hu Q, Feigenbaum GS, Margitich IS, Mazhari R, Boyle AJ, Zambrano JP, Rodriguez JE, Dulce R, Pattany PM, Valdes D, Revilla C, Heldman AW, McNiece I, Hare JM: Bone marrow mesenchymal stem cells stimulate cardiac stem cell proliferation and differentiation. Circ Res 2010;107:913-922.

- 14 Williams AR, Hatzistergos KE, Addicott B, McCall F, Carvalho D, Suncion V, Morales AR, Da Silva J, Sussman MA, Heldman AW, Hare JM: Enhanced effect of combining human cardiac stem cells and bone marrow mesenchymal stem cells to reduce infarct size and to restore cardiac function after myocardial infarction. Circulation 2013;127:213-223.

15 Min JY, Sullivan MF, Yang Y, Zhang JP, Converso KL, Morgan JP, Xiao YF: Significant improvement of heart function by cotransplantation of human mesenchymal stem cells and fetal cardiomyocytes in postinfarcted pigs. Ann Thorac Surg 2002;74:1568-1575.

16 Rubach M, Adelmann R, Haustein M, Drey F, Pfannkuche K, Xiao B, Koester A, Udink ten Cate FE, Choi YH, Neef K, Fatima A, Hannes T, Pillekamp F, Hescheler J, Saric T, Brockmeier K, Khalil M: Mesenchymal stem cells and their conditioned medium improve integration of purified induced pluripotent stem cell-derived cardiomyocyte clusters into myocardial tissue. Stem Cells Dev 2014;23:643-653.

- 17 Spaggiari GM, Capobianco A, Abdelrazik H, Becchetti F, Mingari MC, Moretta L: Mesenchymal stem cells inhibit natural killer-cell proliferation, cytotoxicity, and cytokine production: role of indoleamine 2,3-dioxygenase and prostaglandin E2. Blood 2008;111:1327-1333.

- 18 Zhao S, Wehner R, Bornhauser M, Wassmuth R, Bachmann M, Schmitz M: Immunomodulatory properties of mesenchymal stromal cells and their therapeutic consequences for immune-mediated disorders. Stem Cells Dev 2010;19:607-614.

- 19 Figeac F, Lesault PF, Le Coz O, Damy T, Souktani R, Trebeau C, Schmitt A, Ribot J, Mounier R, Guguin A, Manier C, Surenaud M, Hittinger L, Dubois-Rande JL, Rodriguez AM: Nanotubular crosstalk with distressed cardiomyocytes stimulates the paracrine repair function of mesenchymal stem cells. Stem Cells 2014;32:216-230.

20 Liu L, Jin X, Hu CF, Li R, Zhou Z, Shen CX: Exosomes Derived from Mesenchymal Stem Cells Rescue Myocardial Ischaemia/Reperfusion Injury by Inducing Cardiomyocyte Autophagy Via AMPK and Akt Pathways. Cell Physiol Biochem 2017;43:52-68.

- 21 Kolossov E, Bostani T, Roell W, Breitbach M, Pillekamp F, Nygren JM, Sasse P, Rubenchik O, Fries JW, Wenzel D, Geisen C, Xia Y, Lu Z, Duan Y, Kettenhofen R, Jovinge S, Bloch W, Bohlen H, Welz A, Hescheler J, et al.: Engraftment of engineered ES cell-derived cardiomyocytes but not BM cells restores contractile function to the infarcted myocardium. J Exp Med 2006;203:2315-2327.

- 22 Lepperhof V, Polchynski O, Kruttwig K, Bruggemann C, Neef K, Drey F, Zheng Y, Ackermann JP, Choi YH, Wunderlich TF, Hoehn M, Hescheler J, Saric T: Bioluminescent imaging of genetically selected induced pluripotent stem cell-derived cardiomyocytes after transplantation into infarcted heart of syngeneic recipients. PLoS One 2014;9:e107363.

23 Pfannkuche K, Neuss S, Pillekamp F, Frenzel LP, Attia W, Hannes T, Salber J, Hoss M, Zenke M, Fleischmann BK, Hescheler J, Saric T: Fibroblasts facilitate the engraftment of embryonic stem cell-derived cardiomyocytes on three-dimensional collagen matrices and aggregation in hanging drops. Stem Cells Dev 2010;19:1589-1599.

24 Fatima A, Xu G, Nguemo F, Kuzmenkin A, Burkert K, Hescheler J, Saric T: Murine transgenic iPS cell line for monitoring and selection of cardiomyocytes. Stem Cell Res 2016;17:266-272.

25 Heras-Bautista CO, Katsen-Globa A, Schloerer NE, Dieluweit S, Abd El Aziz OM, Peinkofer G, Attia WA, Khalil M, Brockmeier K, Hescheler J, Pfannkuche K: The influence of physiological matrix conditions on permanent culture of induced pluripotent stem cell-derived cardiomyocytes. Biomaterials 2014;35:73747385.

26 Halbach M, Pfannkuche K, Pillekamp F, Ziomka A, Hannes T, Reppel M, Hescheler J, Muller-Ehmsen J: Electrophysiological maturation and integration of murine fetal cardiomyocytes after transplantation. Circ Res 2007;101:484-492. 


\section{Cellular Physiology Cell Physiol Biochem 2019;52:1309-1324

 \\ Sahito et al.: Cardiomyocyte Transplantation}

27 Muller-Ehmsen J, Peterson KL, Kedes L, Whittaker P, Dow JS, Long TI, Laird PW, Kloner RA: Rebuilding a damaged heart: long-term survival of transplanted neonatal rat cardiomyocytes after myocardial infarction and effect on cardiac function. Circulation 2002;105:1720-1726.

28 Krausgrill B, Vantler M, Burst V, Raths M, Halbach M, Frank K, Schynkowski S, Schenk K, Hescheler J, Rosenkranz S, Muller-Ehmsen J: Influence of cell treatment with PDGF-BB and reperfusion on cardiac persistence of mononuclear and mesenchymal bone marrow cells after transplantation into acute myocardial infarction in rats. Cell Transplant 2009;18:847-853.

29 Suto EG, Mabuchi Y, Suzuki N, Suzuki K, Ogata Y, Taguchi M, Muneta T, Sekiya I, Akazawa C: Prospectively isolated mesenchymal stem/stromal cells are enriched in the CD73(+) population and exhibit efficacy after transplantation. Sci Rep 2017;7:4838.

30 Peister A, Mellad JA, Larson BL, Hall BM, Gibson LF, Prockop DJ: Adult stem cells from bone marrow (MSCs) isolated from different strains of inbred mice vary in surface epitopes, rates of proliferation, and differentiation potential. Blood 2004;103:1662-1668.

31 Cheng K, Li TS, Malliaras K, Davis DR, Zhang Y, Marban E: Magnetic targeting enhances engraftment and functional benefit of iron-labeled cardiosphere-derived cells in myocardial infarction. Circ Res 2010;106:1570-1581.

32 Cheng K, Malliaras K, Li TS, Sun B, Houde C, Galang G, Smith J, Matsushita N, Marban E: Magnetic enhancement of cell retention, engraftment, and functional benefit after intracoronary delivery of cardiacderived stem cells in a rat model of ischemia/reperfusion. Cell Transplant 2012;21:1121-1135.

33 Vandergriff AC, Hensley TM, Henry ET, Shen D, Anthony S, Zhang J, Cheng K: Magnetic targeting of cardiosphere-derived stem cells with ferumoxytol nanoparticles for treating rats with myocardial infarction. Biomaterials 2014;35:8528-8539.

34 Ottersbach A, Mykhaylyk O, Heidsieck A, Eberbeck D, Rieck S, Zimmermann K, Breitbach M, Engelbrecht B, Brugmann T, Hesse M, Welz A, Sasse P, Wenzel D, Plank C, Gleich B, Holzel M, Bloch W, Pfeifer A, Fleischmann BK, Roell W: Improved heart repair upon myocardial infarction: Combination of magnetic nanoparticles and tailored magnets strongly increases engraftment of myocytes. Biomaterials 2018;155:176-190.

35 Moon SH, Ju J, Park SJ, Bae D, Chung HM, Lee SH: Optimizing human embryonic stem cells differentiation efficiency by screening size-tunable homogenous embryoid bodies. Biomaterials 2014;35:5987-5997. 